\title{
The Offspring of Women with Severe Mental Disorder
}

\author{
V.A. Morgan a,b $\cdot$ J. McGrath ${ }^{d} \cdot$ C.M. Hultmane - S. Zubrick $\cdot$ C. Bower ${ }^{c}$. \\ M. Croft ${ }^{\mathrm{a}} \cdot$ G. Valuria $\cdot$ A.V. Jablensky ${ }^{\mathrm{a}, \mathrm{b}}$
}

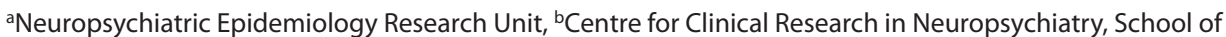
Psychiatry and Clinical Neurosciences, Telethon Institute for Child Health Research, Centre for Child Health Research, The University of Western Australia, Perth, W.A., ${ }^{d}$ Queensland Centre for Mental Health Research and

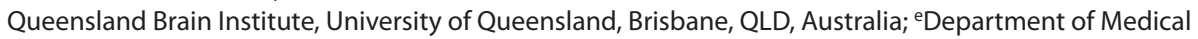
Epidemiology and Biostatistics, Karolinska Institutet, Stockholm, Sweden

Schizophrenia is a severely disabling disorder with a lifetime risk of about $1 \%$. The aetiology of schizophrenia is complex. Evidence from twin, adoptive and family studies indicates that the disorder involves a significant genetic contribution: children of women with schizophrenia have an 8 - to 10 -fold higher risk of developing the disorder compared with the general population [1], with the risk in a monozygotic co-twin increased to about 50-fold [2]. The pattern of inheritance in schizophrenia is not a simple Mendelian one. It is likely that multiple genes of small to moderate effect contribute to the disorder, and that environmental risk factors interact with this genetic susceptibility. However, the precise nature of the genetic and environmental risk factors remains unclear.

It has long been suspected that disruptions to early brain development may be associated with an increased risk of adult-onset schizophrenia [3]. In the 1980s, these speculations became more clearly articulated under the generic label of the 'neurodevelopmental hypothesis' of schizophrenia [4]. It was proposed that critical circuits in the brain were affected in early development by a disease process, with full-blown consequences evident many years later in adolescence or early adulthood as schizophrenia [5]. The original theory is open as to whether the 'disease process' affecting neuronal development is genetically or environmentally determined. There are a number of indicators of neurodevelopmental disruption in schizophrenia including: (a) an increased risk of schizophrenia following obstetric complications at birth with good evidence that the risk is increased 2- to 7-fold [6]; (b) a history of neurointegrative defect in children who later develop schizophrenia [7], and (c) an increased frequency of minor physical anomalies [8] and neurological soft signs [9] in affected 
persons, indicative of a neurodevelopmental disturbance. In light of this, there continues to be considerable interest in understanding schizophrenia as a disorder of neurodevelopment, with a strong emphasis on the role of obstetric complications. In particular, pathological mechanisms that may interfere with neuronal migration during fetal development have been targeted including adverse hypoxic-ischaemic events [6], those affecting immune responses [10] and maternal undernutrition [11]. In more recent years, this line of schizophrenia research has converged with research under the rubric of developmental origins of health and disease [12].

The three studies that follow illustrate some of the work currently in progress in the area of neurodevelopment and schizophrenia. Studies 1 and 2 are record linkage studies, using population-based registers in Western Australia and Sweden, respectively, to follow up outcomes for the children of mothers with schizophrenia. Study 1 has a focus on the relationship between genetic and environmental (obstetric) risk factors and adverse outcomes for these children. The data presented here describe a range of adverse obstetric and offspring outcomes in the perinatal period, including the risk of birth defects. Study 2 also looks at outcomes for the children of mothers with schizophrenia, and is specifically interested in fetal growth and neonatal death and the impact of maternal risk factors such as exposure to smoking on offspring outcomes. In addition, it broadens its scope to include fathers with schizophrenia in the analysis. Finally, study 3 is concerned with schizophrenia as an outcome, rather than an exposure. It takes a neurobiological approach in order to understand better the role of one candidate risk factor for schizophrenia, prenatal exposure to low maternal vitamin D.

\section{Study 1. The Neurodevelopmental Hypothesis and Schizophrenia: Evidence from the Western Australian Study of Women with Severe Mental Illness}

The first study comes from a team of researchers in the Neuropsychiatric Epidemiology Research Unit within the School of Psychiatry and Clinical Neurosciences at the University of Western Australia, working under the direction of Prof. Assen Jablensky. This study is designed to untangle genetic and environmental contributions to the risk for schizophrenia and other adverse outcomes in the children of mothers with schizophrenia and other severe mental illness using Western Australian whole-ofpopulation health databases. Records for 79,599 women on the Western Australian psychiatric case register were cross-linked to 308,022 birth records on the midwives database. Women with psychosis who had given birth in Western Australia between 1980 and 1992 were identified. Comparison mothers were randomly selected from mothers with no record of psychiatric illness. The study database comprised a total of 3,662 mothers and 6,303 children: 382 mothers with schizophrenia (618 children); 763 mothers with bipolar disorder (1,301 children); 686 mothers with unipolar major depression (1,255 children), and 1,831 comparison mothers (3,129 children). 
Table 1. Selected obstetric complications (odds ratio, $95 \%$ confidence interval)

\begin{tabular}{|c|c|c|c|}
\hline & \multicolumn{3}{|l|}{ Maternal diagnosis } \\
\hline & $\begin{array}{l}\text { Schizophrenia vs. } \\
\text { comparison }\end{array}$ & $\begin{array}{l}\text { Bipolar vs. } \\
\text { comparison }\end{array}$ & $\begin{array}{l}\text { Unipolar vs. } \\
\text { comparison }\end{array}$ \\
\hline \multicolumn{4}{|c|}{ Maternal socio-demographic characteristics } \\
\hline Age at delivery $\leq 19$ & $1.5(1.0-2.2)$ & NS & NS \\
\hline Age at delivery $\geq 35$ & $2.0(1.4-2.7)$ & NS & $1.3(1.0-1.7)$ \\
\hline Single/widowed/divorced & $4.3(3.4-5.5)$ & $2.1(1.7-2.6)$ & $1.5(1.2-2.0)$ \\
\hline Partner unemployed/pensioner & $6.0(2.2-16.9)$ & NS & NS \\
\hline Aboriginal & $2.7(1.7-4.2)$ & NS & NS \\
\hline \multicolumn{4}{|l|}{ Pregnancy complications } \\
\hline $\begin{array}{l}\text { Composite scale score }{ }^{1} \text { : pregnancy } \\
\text { complications }\end{array}$ & $1.4(1.2-1.8)$ & $1.2(1.1-1.4)$ & $1.2(1.0-1.4)$ \\
\hline \multicolumn{4}{|l|}{ Specific pregnancy complications } \\
\hline Placenta praevia & NS & $2.0(1.1-3.7)$ & NS \\
\hline Abruption of the placenta & $2.8(1.3-5.7)$ & NS & NS \\
\hline Other antepartum haemorrhage & $1.6(1.0-2.7)$ & $1.7(1.2-2.4)$ & NS \\
\hline Side effects of street drugs & $3.8(1.1-12.8)$ & $3.9(1.5-10.6)$ & NS \\
\hline \multicolumn{4}{|l|}{ Labour/delivery complications (maternal) } \\
\hline $\begin{array}{l}\text { Composite scale score }{ }^{1} \text { : labour and } \\
\text { delivery complications }\end{array}$ & NS & NS & NS \\
\hline \multicolumn{4}{|l|}{ Neonatal complications } \\
\hline $\begin{array}{l}\text { Composite scale score }{ }^{1} \text { : neonatal } \\
\text { complications }\end{array}$ & $1.3(1.0-1.5)$ & NS & NS \\
\hline \multicolumn{4}{|l|}{ Specific neonatal complications } \\
\hline Fetal distress & $1.4(1.1-1.8)$ & NS & NS \\
\hline Narcotic antagonist used & $1.9(1.2-2.9)$ & NS & $1.6(1.1-2.3)$ \\
\hline $\begin{array}{l}\text { Percent of expected birthweight } \\
<10 \text { th percentile }\end{array}$ & $1.4(1.0-1.9)$ & NS & NS \\
\hline \multicolumn{4}{|l|}{ Birth defects } \\
\hline Any birth defects & NS & NS & NS \\
\hline Cardiovascular defects & $2.6(1.2-5.5)$ & NS & NS \\
\hline $\begin{array}{l}\text { Other defects (mainly minor physical } \\
\text { anomalies) }\end{array}$ & $2.2(1.1-4.5)$ & NS & NS \\
\hline
\end{tabular}

NS = Not significant.

${ }^{1}$ McNeil-Sjöström Scale for measuring obstetric complications. Severity level 4 [14, 15]. 
Fathers were identified using birth registrations. Full psychiatric histories for mothers, fathers and children were extracted, and data collected on obstetric complications and other childhood morbidities [for a more detailed description of the study design, see Jablensky et al. 13]. The study aims were to: (a) determine the frequency and distribution of obstetric complications in women with schizophrenia compared with a non-psychiatric comparison group of mothers; (b) explore the spectrum of outcomes for the children born to women with schizophrenia; (c) assess specificity of findings to maternal schizophrenia compared with maternal bipolar disorder and unipolar major depression, and (d) examine the relationship between familial psychiatric status, obstetric complications and mental health outcomes for children (in work in progress). The main study findings to date are summarised in table 1.

The Western Australian researchers found that mothers in all three diagnostic groups were more likely than comparison women to be single mothers, and to experience socioeconomic disadvantage relative to the general population. In addition, women with schizophrenia were characterized by a significant excess of either very young (age $\leq 19$ ) or older (age $\geq 35$ ) mothers. They were also more likely to have partners who were unemployed or disabled.

With respect to obstetric complications, this research team found that crude odds ratios (ORs) calculated using the McNeil-Sjöström summated scores indicated that mothers in all three diagnostic groups were significantly more likely to have complications during the pregnancy period. The risk of labour and delivery complications was not increased for any diagnostic group, while the risk of neonatal complications was increased only in offspring of women with schizophrenia. Crude ORs were also calculated for specific obstetric complications. Women with schizophrenia and bipolar disorder, but not women with unipolar depression, were significantly more likely to experience placental abnormalities, antepartum haemorrhages, and toxic side effects of drugs (alcohol, tobacco and illicit substances) compared with unaffected women. Children in all three diagnostic groups were more likely to experience fetal distress (significant in offspring of schizophrenia mothers), and to have a narcotic antagonist administered (significant in mothers with schizophrenia and unipolar depression). There was a non-significant tendency for a suboptimal 5-min Apgar score in the offspring of the women with schizophrenia, as well as for delayed respiration and intubation. There were no significant differences among the groups in mean gestational age, but newborns of the mothers with schizophrenia had a lower mean birthweight $(3,248 \mathrm{~g}$ versus $3,334 \mathrm{~g}$ in the comparison group), a smaller head circumference (11.4\% were in the lowest 10 th percentile compared with $9.5 \%$ of the comparison offspring), and tended to be shorter (9.4\% in the lowest 10 th percentile compared with $8.7 \%$ of the offspring of unaffected mothers). A significantly larger proportion of babies of the mothers with schizophrenia (12.6\% compared with $9.3 \%$ of the comparison group, $10.4 \%$ of the offspring of the mothers with unipolar disorder, and $9.9 \%$ of the offspring of the women with bipolar disorder) fell into the lowest 10th percentile of intrauterine growth using an index of percentage of expected birthweight based on 
whole-of-population birth data for Western Australia [16]. Smoking is known to have a marked impact on infant birthweight. When smoking in pregnancy was modelled, even assuming a worst-case scenario based on smoking data from the Australian national epidemiological survey of psychosis [17], it was not possible to explain all of the birthweight reduction in the offspring of mothers with schizophrenia. Finally, the risk of having any birth defect was similar for all children, regardless of their mother's psychiatric status. However, two categories of birth defects, cardiovascular defects (ventricular and atrial septal defects, patent ductus arteriosus, anomalies of the aorta) and minor physical anomalies, were significantly elevated in the children of women with schizophrenia.

Some $45 \%$ of the case children were born before their mother's first psychiatric admission. Multivariate analyses indicated that, relative to the comparison sample, mothers whose psychiatric illness commenced before the birth of their child were significantly more likely to experience obstetric complications (adjusted OR 1.1, 95\% confidence interval, CI, 1.1-1.2). By contrast, mothers whose psychiatric illness commenced after the birth of their child were at no greater risk of obstetric complications than comparison mothers. However, there were a number of individual complications, specific to mothers with schizophrenia, where both pre- and post-onset rates were elevated. These included: abruption of the placenta; low birthweight; cardiovascular defects, and minor physical anomalies.

\section{Summary}

This study found that women with schizophrenia, bipolar disorder, and unipolar depression experience an increased overall incidence of obstetric complications, relative to the non-psychiatric comparison group. A major factor contributing to the increased risk of obstetric complications in women with schizophrenia and, to a lesser degree, in women with affective disorders appears to be the clustering of adverse maternal characteristics. Moreover, it is likely that behavioural disorganisation and environmental exposures including poor nutrition and substance use may play an important role in the excess of obstetric complications in births that occur after the onset of psychosis only. This is supported by the finding that the incidence of adverse outcomes in all three diagnostic groups was significantly increased only in pregnancies occurring after the onset of psychiatric illness. Some obstetric complications showed no pre-onset/post-onset differences. These occurred only in women with schizophrenia, and included placentation abnormalities, low birthweight, minor physical anomalies and cardiovascular birth defects. This constancy in rates suggests a diagnosis-specific pre-existing susceptibility that may involve both genetic and environmental components. The excess of cardiovascular birth defects, specific to mothers with schizophrenia, may involve parentally transmitted genes, some of which may be expressed in both the heart and brain or are in linkage disequilibrium 
with other genes conferring susceptibility to schizophrenia. It is also conjectured that low birthweight, occurring only in mothers with schizophrenia and with increased frequency in southern hemisphere springtime, may point to maternal exposure to winter infection resulting in a compromised intrauterine environment and restricted fetal growth.

\section{Study 2. Adverse Pregnancy Outcome in Schizophrenia: Maternal and Paternal Influences}

Prof. Christina Hultman and her team of researchers in the Department of Medical Epidemiology and Biostatistics at the Karolinska Institutet in Sweden are also working on pregnancy outcomes for women with schizophrenia. These women face an increased risk of adverse pregnancy outcomes: many smoke, misuse other substances and are socio-economically disadvantaged. Not only are these variables well-known risk factors for adverse pregnancy outcomes, but smoking is known to be causally related to fetal growth retardation and is also possibly associated with stillbirth and preterm birth. In several recent population-based studies, the increased risks for preterm birth, low birth weight and infant death in women with schizophrenia remained even after controlling for these covariates. Nonetheless, Prof. Hultman's research has previously shown that the increased risk for preterm birth among women with schizophrenia decreased from 70 to $40 \%$ when controlling for maternal factors [18]. Women who had had an episode of schizophrenia during their pregnancy had the highest risk of (a) preterm delivery (delivery before the 37th gestational week; OR 3.4, 95\% CI 2.1-5.4), (b) low birth weight (birthweight under 2,500 g; OR 4.3, 95\% CI 2.9-6.6), and (c) stillbirth (OR 4.4, 95\% CI 1.4-13.8). Controlling for the high incidence of smoking during pregnancy among women with schizophrenia (51 vs. $24 \%$ in the normal population) and other maternal factors (single motherhood, maternal age, parity, maternal education, mothers' country of birth and pregnancy-induced hypertensive diseases) in a multiple regression model reduced the risk estimates markedly. However, even after adjustment, the risk for adverse pregnancy outcomes was generally doubled for women with an episode of schizophrenia during pregnancy compared with women in the control group. To test the hypothesis that infants of women with affective psychosis face increased risks of adverse pregnancy outcomes, Prof. Hultman and colleagues performed a similar study with data from the Swedish Medical Birth Register and the Hospital Discharge Register [19] on 5,593 births to mothers with affective psychoses and 46,068 control mothers. Births to mothers with affective psychosis had an increased risk of giving birth to preterm, small or growth-retarded babies. The risk for stillbirth and infant death during the 1st year of life was not significantly higher. The risks were greatest in mothers receiving hospital treatment for affective disorder during pregnancy $(\mathrm{OR}=2.67,95 \% \mathrm{CI} 1.71-4.17)$. Affected mothers were approximately twice as likely to be heavy smokers than controls in this sample. 
What might be the causes of the higher risk of preterm birth in women experiencing a psychotic episode during pregnancy? The risk may be partly accounted for by the use of antipsychotic medication during pregnancy, or by an increase in antipsychotic dosage during this period [20]. There has been some concern that antipsychotic medication may compromise uterine blood flow, and produce post-partum neonatal sedation and extrapyramidal signs. In a study following up the outcome of 215 pregnancies exposed to haloperidol or penfluridol in the first trimester, a higher rate of preterm births (13.9 vs. $6.9 \%, \mathrm{p}=0.006)$ was found compared with unexposed control pregnancies. The rate of congenital anomalies was not increased, but there were indications of fetal growth restriction, with a lower median birth weight for exposed full-term infants ( 3,250 vs. $3,415 \mathrm{~g}, \mathrm{p}=0.004)$. A second explanation may be that women with schizophrenia attend antenatal care clinics less frequently, and therefore may suffer from underdiagnosed medical conditions that affect their pregnancy outcomes. A third possibility is a common familial vulnerability for nonoptimal pregnancy outcomes. Schizophrenia is under a high degree of genetic influence and offspring carry a 10\% risk of developing schizophrenia themselves.

Less is known about the offspring's risk for adverse pregnancy outcomes if the father has schizophrenia. A recent study by Webb and colleagues found an increased risk for post-neonatal death among offspring of fathers with schizophrenia [21]. However, the risk was not statistically significant and a meta-analysis by Webb and colleagues indicated a need for more evidence on the effects of exposure to paternal disorder. Recent data based on a cohort of 1,890,550 births in Sweden indicate that, as well as offspring of mothers with schizophrenia, offspring of fathers with schizophrenia have an increased risk of non-optimal birth outcomes. It was shown that offspring of fathers with schizophrenia have increased risks for infant death, low birth weight, and being small for gestational age [22]. It is likely then that paternal schizophrenia also confers a risk for adverse pregnancy outcomes. Furthermore, since the risks were attenuated when covariates were included in the models, it is also possible to conclude that adverse pregnancy outcomes were not a consequence of schizophrenia in the parents, but rather a consequence of adverse socio-environmental conditions. However, the association between both maternal and paternal schizophrenia and infant death remained after controlling for these covariates. This could be attributed to environmental causes, but it is also possible that it is due to a common genetic effect.

In order to explore further the contribution of genetic factors to the associations reported, the Karolinska team studied the risk for adverse pregnancy outcomes and infant death among the full- and half-siblings of parents with schizophrenia. A genetic factor would be indicated if the risk was significantly higher for parental full siblings compared with parental half-siblings. Using the Swedish Multigeneration Register, we linked family information with the Swedish Hospital Discharge Register. For both siblings of mothers and siblings of fathers, there seems to be a difference in outcomes for full sisters compared with half-sisters. Thus, the researchers found a modest, but significantly increased risk of low birth weight if the parental full sibling 
had schizophrenia but not if the parental half-sibling had the disease, suggesting that, in addition to a maternal effect, there was a genetic mediation of the association between schizophrenia and low birthweight. On the other hand, the risk for infant death was not increased if the parent had a sibling with schizophrenia.

\section{Summary}

Not only do offspring of mothers with schizophrenia have an increased risk for non-optimal birth outcomes, but offspring of fathers with schizophrenia also are at increased risk for adverse outcomes. Much of the increased risk can be explained by maternal psychosocial factors such as smoking and single motherhood. In addition to non-optimal birth outcomes, the offspring of mothers and of fathers with schizophrenia also have double the risk of infant mortality. Excess mortality is largely attributable to post-neonatal death, but not sudden infant death syndrome, and cannot be explained by maternal behaviour during pregnancy. Consequently, it is important for researchers to focus on the mechanisms underlying these associations. This would include an examination of the effects of psychotropic medication on the developing fetus and the further work on the role of genetic risk factors. While a genetic risk factor may be implicated in the findings for birth weight, it does not appear to be indicated with respect to the findings for infant mortality.

\section{Study 3. Linking Schizophrenia Epidemiology and Developmental Neurobiology: The Impact of Low Prenatal Vitamin D on Brain Development}

It has been hypothesised by a research group led by Prof. John McGrath at The Queensland Centre for Mental Health Research and the Queensland Brain Institute, University of Queensland, that low prenatal vitamin D may be a risk factor for schizophrenia [23]. Many studies have shown that children born in winter and spring have a significantly increased risk of developing schizophrenia in later life [24]. Children born at higher latitudes are also at increased risk [25], with both the incidence and prevalence of schizophrenia being significantly greater in sites from higher latitudes [26]. Of interest, data from cold climates indicate that the incidence of schizophrenia is significantly higher in dark-skinned migrants compared with the native born [27]. Given that hypovitaminosis D is more common (a) during winter and spring, (b) at high latitudes, and (c) in dark-skinned individuals [28], low prenatal vitamin D 'fits' these key environmental features. Preliminary evidence from analytical epidemiology studies also suggests that prenatal vitamin D warrants closer attention as a candidate risk factor. For example, vitamin D supplements in the 1st year of life significantly reduced the risk of schizophrenia in males in a large Finnish Birth Cohort [29]. In addition, 25-hydroxyvitamin $\mathrm{D}_{3}$ serum levels in 26 mothers whose children 
developed schizophrenia were numerically (but not significantly) lower than that of 51 control mothers whose children did not develop the disease [30]. There was a trend-level association between low maternal vitamin D levels and schizophrenia in a subgroup of children of African-American mothers [30], who would be at greatest risk of vitamin $\mathrm{D}$ deficiency because of their increased skin pigmentation. Access to larger samples of archival maternal and neonatal sera will be required to examine this hypothesis adequately. However, banked prenatal and/or neonatal samples of individuals who have developed schizophrenia are rare resources. Even if such samples were available, the ability to interpret associations from observational studies is an ever-present problem for epidemiology [31]. Randomized control trials of adverse prenatal exposures are clearly not an option. In the absence of alternative strategies, schizophrenia epidemiology is prone to become trapped in cycles of uninformative replications ('circular epidemiology') [32].

While analytical epidemiology is still a critical research tool, various types of 'translational epidemiology' can provide complementary research strategies. Linking exposures with genetic polymorphisms in genes that impact on relevant pathways is one such option [33]. Creating strong links between schizophrenia epidemiology and neuroscience is also a valuable research strategy. It can provide biological plausibility to candidate exposures, which can then feedback to refine measures of exposure or related research designs. Furthermore, the exploration of clues from epidemiology based on molecular, cellular and behavioural neuroscience can act as a catalyst for neuroscience discovery.

In the last few years, Prof. McGrath's group has been testing the hypothesis that prenatal vitamin $\mathrm{D}$ deficiency alters brain development and adult behaviour in rodent models. The experiments involve depleting female rats of vitamin D, mating them, and then repleting the dams with vitamin $\mathrm{D}$ immediately after birth. The offspring have been examined as neonates and as adults. The developmental vitamin D deficiency model (abbreviated as DVD model), is a developmental exposure only: from birth onwards, all maternal animals receive a diet containing normal levels of vitamin D. Full details of the dietary and breeding program are provided elsewhere [34].

The evidence shows that DVD deficiency leads to physiological alterations in pups at birth and into adulthood. DVD-deficient neonates had larger lateral ventricles, increased cell proliferation and reduced apoptosis, reduced density of neurotrophin receptor $\left(\mathrm{p} 75^{\mathrm{NTR}}\right)$ and reduced levels of nerve growth factor and glial cell line-derived growth factor compared with controls $[35,36]$. As adults, these animals had larger lateral ventricles and reduced nerve growth factor expression compared with controls [37]. DVD-deficient adults had significant changes in protein and gene expression in the brain $[38,39]$. From a behavioural perspective, DVD-deficient adults were more active than controls (that is, showing hyperlocomotion) [34, 40], and had altered attentional processing indicated by impaired latent inhibition [41]. Finally, some of the most robust and consistent findings in the DVD model have emerged in pharmacological studies. For example, the research group has shown that DVD-deficient rats have enhanced locomotion in response to the NMDA antagonist MK-801; a 
behaviour which is blocked by the neuroleptic haloperidol [34]. More recently, the DVD model has also been established in the mouse [42].

\section{Summary}

The animal experimental data have provided strong evidence demonstrating that DVD deficiency during gestation alters the trajectory of brain development in rodent models. Of particular interest are features of the DVD phenotype that are informative for schizophrenia research. Most notably, the increase in lateral ventricular volume is one of the most consistent neurobiological correlates of schizophrenia [43] and heightened sensitivity to dopaminergic and glutamatergic agents, which are neurotransmitters that are both thought to function abnormally in the brains of patients $[44,45]$. Because hypovitaminosis $\mathrm{D}$ is prevalent in pregnant women [46], this research may have important public health implications. While the epidemiological evidence linking low prenatal vitamin $\mathrm{D}$ and schizophrenia remains inconclusive, the animal experiments have provided compelling evidence about the role of vitamin $\mathrm{D}$ in brain development. Thus, clues from schizophrenia epidemiology have uncovered previously unsuspected pathways involving vitamin $\mathrm{D}$ and brain development.

\section{Conclusion}

Exposures in utero and the early neonatal period may have short and long-term neuropsychiatric and other consequences. These risks may operate interactively with or independently of genetic risk for schizophrenia. This chapter outlined several approaches to the study of neurodevelopment and schizophrenia. In one approach, long-standing, prospective population-based health registers in Western Australia and Sweden have been employed as an efficient and cost-effective resource in the study of rare and complex diseases. Their use has helped advance our understanding of genetic and environmental factors associated with adverse obstetric and offspring outcomes in women with schizophrenia. Using a different approach to advance our understanding in this area of research, investigators in Queensland are working together with neuroscientists to evaluate the biological plausibility of candidate exposures [38], in this case vitamin D, using animal models.

Although genetic liability may account for some of the findings, all three research programs provide good evidence that maternal risk factors in pregnancy are also major determinants of adverse outcomes. Policies to ensure that mothers are well supported during their pregnancies so as to reduce risks related to poor outcomes are indicated. This is particularly the case for vulnerable women with severe mental illness. Data presented here go even further and suggest that paternal characteristics and psychopathology are also important in the antenatal period, and more attention needs to be paid to 
fathers during this critical time. The clinical significance of research into neurodevelopment and obstetric pathology in schizophrenia is not trivial. Women make up some $40 \%$ of the prevalent cases of psychosis, and about half the women will have children. More particularly, for the majority, schizophrenia onset is during their peak childbearing years [17]. Through this series of studies, the hope is that we will understand better both the consequences of sub-optimal uterine environments for the offspring of parents with schizophrenia, as well as the impact of neurodevelopmental disruption in utero on the risk of onset of schizophrenia in late adolescence-early adulthood for any child. The capacity to identify modifiable risk factors will not only improve outcomes for mothers and fathers with schizophrenia and their children, but also for the community at large.

\section{References}

1 Gottesman II, McGuffin P, Farmer AE: Clinical genetics as clues to the 'real' genetics of schizophrenia (a decade of modest gains while playing for $>_{13}$ time). Schizophr Bull 1987;13:23-44.

$\checkmark 2$ Gottesman II, Erlenmeyer-Kimling L: Family and twin strategies as a head start in defining prodromes and endophenotypes for hypothetical early-interventions in schizophrenia. Schizophr Res 2001;51: 93-102.

3 Kraepelin E: Dementia Praecox and Paraphrenia (translated by R.M. Barclay). Edinburgh, Livingstone, 1919.

-4 Weinberger DR: Implications of normal brain development for the pathogenesis of schizophrenia. Arch Gen Psychiatry 1987;44:660-669.

5 Marenco S, Weinberger D: The neurodevelopmental hypothesis of schizophrenia: following a trail of evidence from the cradle to the grave. Dev Psychopathol 2000;12:501-527.

6 Cannon M, Jones PB, Murray RM: Obstetric complications and schizophrenia: historical and meta-analytic review. Am J Psychiatry 2002;159:1080-1092.

7 Fish B: Neurobiologic antecedents of schizophrenia in children. Arch Gen Psychiatry 1977;34:12971313.

8 Weinberg SM, Jenkins EA, Marazita ML, Maher BS: Minor physical anomalies in schizophrenia: a metaanalysis. Schizophr Res 2007;89:72-85.

$\checkmark 9$ Ismail B, Cantor-Graae E, McNeil TF: Neurological abnormalities in schizophrenic patients and their siblings. Am J Psychiatry 1998;155:84-89.

10 Brown AS: Prenatal infection as a risk factor for schizophrenia. Schizophr Bull 2006;32:200-202.

-11 Susser E, Neugebauer R, Hoek HW, Brown AS, Lin S, Labovitz D, et al: Schizophrenia after prenatal famine. Further evidence. Arch Gen Psychiatry 1996;53:25-31.
12 Barker DJP: Fetal and infant origins of adult disease. BMJ 1992;301:1111.

3 Jablensky A, Morgan V, Zubrick S, Bower C, Yellachich L: Pregnancy, delivery, and neonatal complications in a population cohort of women with schizophrenia and major affective disorders. Am J Psychiatry 2005;162:79-91.

14 McNeil T, Cantor-Graae E, Sjöström K: Obstetric complications as antecedents of schizophrenia: empirical effects of using different obstetric complication scales. J Psychiatr Res 1994;28:519-530.

15 McNeil TF, Sjöström K: McNeil-Sjöström Scale for Obstetric Complications. Version B: $950301 \mathrm{~b}$. Malmö, Lund University, 1995.

16 Blair E: The undesirable consequences of controlling for birth weight in perinatal epidemiological studies. J Epidemiol Commun Health 1996;50:559563.

17 Jablensky A, McGrath J, Herrman H, Castle D, Gureje O, Morgan V, et al: Psychotic disorders in urban areas: an overview of the methods and findings of the Study on Low Prevalence Disorders, National Survey of Mental Health and Wellbeing 1996-1998. Aust N Z J Psychiatry 2000;34:221-236. 8 Nilsson E, Lichtenstein P, Cnattingius S, Murray R, Hultman C: Women with schizophrenia: pregnancy outcome and infant death among their offspring. Schizophr Res 2002;58:221-229.

19 MacCabe JH, Martinsson L, Lichtenstein P, Nilsson E, Cnattingius S, Murray RM, et al: Adverse pregnancy outcomes in mothers with affective psychosis. Bipolar Disord 2007;9:305-309.

20 Pinkofsky HB: Psychosis during pregnancy: treatment considerations. Ann Clin Psychiatry 1997;9: 175-179. 
21 Webb R, Abel K, Pickles A, et al: Mortality in offspring of parents with psychotic disorders: A critical review and metal-analysis. Am J Psychiatry 2005; 162:1045-1056.

-22 Nilsson E, Hultman CM, Cnattingius S, Otterblad Olausson P, Björk C, Lichtenstein P: Schizophrenia and offspring's risk for adverse pregnancy outcomes and infant death. Br J Psychiatry 2008;193:311-315.

23 McGrath J: Hypothesis: is low prenatal vitamin D a risk-modifying factor for schizophrenia? Schizophr Res 1999;40:173-177.

-24 Torrey EF, Miller J, Rawlings R, Yolken RH Seasonality of births in schizophrenia and bipolar disorder: a review of the literature. Schizophr Res 1997;28:1-38.

25 Saha S, Chant DC, Welham JL, McGrath JJ: The incidence and prevalence of schizophrenia varies with latitude. Acta Psychiatr Scand 2006;114:36-39.

26 Davies G, Welham J, Chant D, Torrey EF, McGrath J: A systematic review and meta-analysis of Northern Hemisphere season of birth studies in schizophrenia. Schizophr Bull 2003;29:587-593.

27 Cantor-Graae E, Selten JP: Schizophrenia and migration: a meta-analysis and review. Am J Psychiatry 2005;162:12-24.

28 Holick MF: Environmental factors that influence the cutaneous production of vitamin D. Am J Clin Nutr 1995;61:638S-645S.

29 McGrath J, Saari K, Hakko H, Jokelainen J, Jones P, Järvelin MR, Chant D, Isohanni M: Vitamin D supplementation during the first year of life and risk of schizophrenia: a Finnish birth cohort study. Schizophr Res 2004;67:237-245.

30 McGrath J, Eyles D, Mowry B, Yolken R, Buka S: Low maternal vitamin $\mathrm{D}$ as a risk factor for schizophrenia: a pilot study using banked sera. Schizophr Res 2003;63:73-78.

-31 Davey Smith G, Ebrahim S: Epidemiology - is it time to call it a day? Int J Epidemiol 2001;30:1-11.

32 Kuller LH: Circular epidemiology. Am J Epidemiol 1999;150:897-903.

33 Smith GD, Ebrahim S: Mendelian randomization: prospects, potentials, and limitations. Int J Epidemiol 2004;33:30-42.

34 Kesby JP, Burne TH, McGrath JJ, Eyles DW: Developmental vitamin D deficiency alters MK 801-induced hyperlocomotion in the adult rat: an animal model of schizophrenia. Biol Psychiatry 2006;60:591-596.
35 Eyles D, Brown J, Mackay-Sim A, McGrath J, Feron F: Vitamin D3 and brain development. Neuroscience 2003;118:641-653.

36 Ko P, Burkert R, McGrath J, Eyles D: Maternal vita$\min \mathrm{D}(3)$ deprivation and the regulation of apoptosis and cell cycle during rat brain development. Brain Res Dev Brain Res 2004;153:61-68.

37 Feron F, Burne TH, Brown J, Smith E, McGrath JJ, Mackay-Sim A, et al: Developmental vitamin D3 deficiency alters the adult rat brain. Brain Res Bull 2005;65:141-148.

38 Almeras L, Eyles D, Benech P, Laffite D, Villard C, Patatian A, et al: Developmental vitamin D deficiency alters brain protein expression in the adult rat: implications for neuropsychiatric disorders. Proteomics 2007;7:769-780.

39 Eyles D, Almeras L, Benech P, Patatian A, MackaySim A, McGrath J, et al: Developmental vitamin D deficiency alters the expression of genes encoding mitochondrial, cytoskeletal and synaptic proteins in the adult rat brain. J Steroid Biochem Mol Biol 2007; 103:538-545.

40 Burne TH, Becker A, Brown J, Eyles DW, MackaySim A, McGrath JJ: Transient prenatal vitamin D deficiency is associated with hyperlocomotion in adult rats. Behav Brain Res 2004;154:549-555.

41 Becker A, Eyles DW, McGrath JJ, Grecksch G: Transient prenatal vitamin D deficiency is associated with subtle alterations in learning and memory functions in adult rats. Behav Brain Res 2005;161: 306-312.

42 Harms LR, Eyles DW, McGrath JJ, Mackay-Sim A, Burne TH: Developmental vitamin D deficiency alters adult behaviour in $129 / \mathrm{SvJ}$ and C57BL/6J mice. Behav Brain Res 2008;187:343-350.

43 Harrison PJ, Weinberger DR: Schizophrenia genes, gene expression, and neuropathology: on the matter of their convergence. Mol Psychiatry 2005;10:4068.

44 Olney JW, Farber NB: Glutamate receptor dysfunction and schizophrenia. Arch Gen Psychiatry 1995;52:998-1007.

45 Laruelle M, Frankle WG, Narendran R, Kegeles LS, Abi-Dargham A: Mechanism of action of antipsychotic drugs: from dopamine $\mathrm{D}(2)$ receptor antagonism to glutamate NMDA facilitation. Clin Ther 2005;27(suppl A):S16-S24.

46 Hollis BW, Wagner CL: Vitamin D deficiency during pregnancy: an ongoing epidemic. Am J Clin Nutr 2006;84:273.

\section{Dr. Vera A. Morgan}

The University of Western Australia School of Psychiatry and Clinical Neurosciences

Level 3 Medical Research Foundation Building

Rear 50 Murray Street, Perth, WA 6000 (Australia)

Tel. +61 89224 0235/0290, Fax +61 89224 0285, E-Mail vmorgan@cyllene.uwa.edu.au 